\title{
Surgical Management of Sacrococcygeal Region Giant Tumors by Use of Balloon Occlusion Abdominal Aorta
}

\author{
Hui S GONG ${ }^{1}$, Gang J LIU², Qing S HUANG², Feng H CHEN', Hao B ZHAO'1 \\ ${ }^{1}$ West China Hospital, Department of Neurosurgery, China \\ ${ }^{2}$ Sichuan University, West China Medical School, China
}

\section{ABSTRACT}

AIM: Sacrococcygeal region giant tumors are a challenge for neurosurgeons. The purpose of this paper was to retrospectively analyze the clinical records of sacrococcygeal region giant tumors treated surgically by balloon occlusion of the abdominal aorta.

MATERIAL and METHODS: A total of 130 patients of sacral region tumors underwent surgery in our department from February 2009 to February 2013. Among these patients, 35 giant tumors were treated by balloon catheter occlusion of the abdominal aorta and electrophysiological monitoring. Thirty patients returned for follow-up evaluations and their clinical and imaging records were analyzed.

RESULTS: Thirty patients underwent surgery via a posterior approach; these cases included 21 chordomas, 5 schwannomas, and 4 giant cell tumors of bone. Wide resections were performed in 26 patients (86.7\%) and margin resections were performed in 4 (13.3\%) patients. Most patients' symptoms were relieved through surgery and only nine patients (30\%) experienced recurrence of the tumors during follow-up.

CONCLUSION: Sacrococcygeal region giant tumors are still difficult to treat, especially for malignant tumors. Balloon catheter occlusions of the lower abdominal aorta can notably decreased intraoperative hemorrhage, shorten operation time, and decrease postoperative complications. This method is a good choice for neurosurgeons to manage these giant tumors in the sacral region.

KEYWORDS: Surgical management, Balloon catheter, Abdominal aorta occlusion, Sacrococcygeal region, Giant tumors

\section{INTRODUCTION}

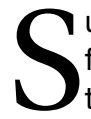
urgical management of large sacral tumors is challenging for surgeons and anesthesiologists, in particular due to extensive hemorrhage, which is often refractory to control strategies $(8,10,17,20)$. Several studies $(7,9,12,15$, $16,18)$ have reported that occluding the abdominal aorta with a balloon catheter is an effective method to control intraoperative blood loss while also gaining better outcomes and fewer complications.

The sacrococcygeal region is a complex anatomical area. The pelvic cavity provides many spaces for tumors to grow to quite a large size before symptoms become obvious (19).
Controlling intraoperative hemorrhage is a very troublesome problem for these giant tumors, but it is of vital significance. When blood loss exceeds the maximum permissive level, it becomes life threatening. The purpose of this study is to report on our successful experience in controlling blood loss through using balloon catheters to occlude the abdominal aorta.

\section{MATERIAL and METHODS}

\section{Patients Characteristics, Inclusion and Exclusion Criteria}

From February 2009 to February 2013, 130 patients with sacral region tumors underwent surgery at the Neurosurgery Department of West China Hospital. Of these, the method of 
balloon catheter occlusion of the abdominal aorta was used in 35 patients. Finally, 30 patients $(23.1 \%)$ met our inclusion criteria and were included for analysis in this study. 17 females and 13 males ranging in age from 21 to 79 years (mean, 55.2). Among these patients, there were 21 chordomas, 5 schwannomas, and 4 giant cell tumors of bone. Twentytwo of the patients experienced sacrococcygeal pain, 10 patients complained of urinary or bowel dysfunction, and 7 patients presented with lower limb numbness. Patients that matched the following criteria were considered: (1) diagnosis of sacrococcygeal giant tumors (maximum tumor diameter $>6 \mathrm{~cm}$ ); (2) surgical treatment combined with abdominal aorta occlusion and electrophysiological monitoring; and (3) complete clinical records and follow-up information. Cases in which there were small tumors, incomplete clinical records, no use of balloon occlusion of the abdominal aorta, and lack of follow-up data were not considered. The basic information of the 30 patients is listed in Table I.

\section{Preoperative Imaging Examinations and Assessment}

Sacrococcygeal enhanced magnetic resonance imaging (MRI) scans, three-dimensional computed tomography (CT) scans, and angiography were performed in all 30 patients. MRI was used to diagnose the tumors and define the relationship with the surrounding soft tissues, while 3D-CT was performed to observe bone invasion more directly. Detailed imaging findings are presented in Tables I and II. Tumor size was estimated in each case by the maximum tumor diameter. The mean maximum tumor diameter was $9.7 \mathrm{~cm}$ (range, 7 to 15 $\mathrm{cm}$ ). The upper extent of the lesion was at S3 or the proximal part in $20(66.7 \%)$ patients and the upper part was located at $\mathrm{S} 4$ or distal end in patients 10 (33.3\%). Angiography was used to exclude vascular diseases and to place the balloon catheter via the right femoral artery (Figure $1 \mathrm{~A}$ ).

\section{Operative Techniques}

The percutaneous coronary intervention sheath was inserted into the abdominal aorta via the femoral artery in the intervention operating room. After measuring the distance between the access point and the umbilicus, the balloon dilation catheter was inserted into the percutaneous coronary intervention sheath to reach the abdominal aorta and was retained at the site between the renal artery branch and the common iliac artery branch with the assistance of digital subtraction angiography (DSA) monitoring (15), the balloon was always retained in the L3-4 level (Figure 1B). Then patients were sent to the surgical operation room. All patients were placed in the prone position following general anesthesia and intubation. The electrodes of the electrophysiological monitor were inserted in the anal sphincter and bilateral lower limb muscle to detect bowel and lower limb motor function. A "comma" incision was marked according to the preoperative imaging findings (Figure 2A). A solution of $10 \mathrm{ml}$ of heparin (5 $\mathrm{U} / \mathrm{ml}$ ) or more was filled in the balloon catheter until the lower abdominal aorta was occluded completely (confirmed by zero oxygen saturation of the toes bilaterally and the disappearance of the pulse of the bilateral dorsal artery). The next step was to exposure the tumor and wide resected. The upper portion of the tumor was usually exposed and resected first (Figure $2 \mathrm{~B}$ ), then gradually moved to the surround until it was beyond the margin of tumor (more than $1 \mathrm{~cm}$ ), including the erosion sacrococcygeal being resected together. When permitted, electrophysiological monitoring was used to carefully protect the integrity of the sacral nerves. Gelfoam was used to fill the interstitial space. Silicone tubes and vascular compression devices were used in all patients postoperatively. Since we adopted the use of the balloon occlusion technique applied to the lower abdominal aorta, intraoperative hemorrhage and surgical time have dramatically decreased. The mean blood
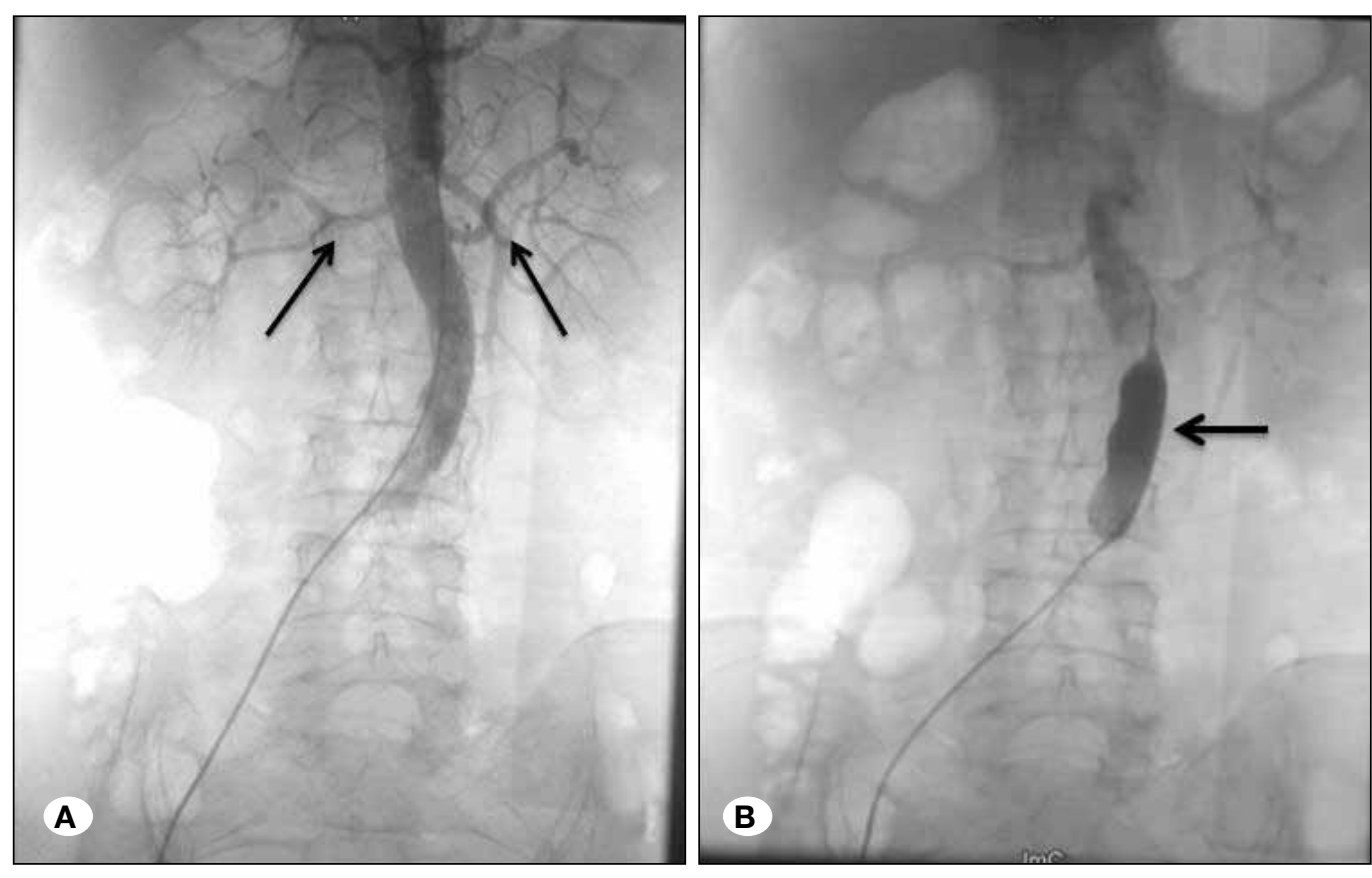

Figure 1: The black arrowhead means the bilateral renal artery (A) and the balloon retains in L3-4 level (B). 
loss was $224.0 \mathrm{ml}$, the mean operation time was 107.1 minutes, and the mean occlusion time was 69 minutes (Table II).

\section{Follow-Up Assessments}

Thirty patients were available for follow-up assessment 6 to 60 months; mean, 29.0). Enhanced MRI scan had been performed three months postoperatively and each year after surgery to detect tumor recurrence. All patients received radiotherapy at least one time postoperatively. Clinical symptoms were classified into three different degrees (improved, same, and worse) and these symptoms were assessed at the last followup measure.

Table I: The Basic Information of 30 Patients

\begin{tabular}{|c|c|c|c|c|c|c|c|}
\hline $\begin{array}{l}\text { Patient } \\
\text { No }\end{array}$ & $\begin{array}{c}\text { Age } \\
\text { (years) }\end{array}$ & Sex & Upper level & $\begin{array}{c}\text { Tumor max } \\
\text { diameter }(\mathbf{c m})\end{array}$ & $\begin{array}{c}\text { Operation } \\
\text { time (minutes) }\end{array}$ & $\begin{array}{l}\text { Blood } \\
\text { loss (ml) }\end{array}$ & $\begin{array}{c}\text { Follow-up } \\
\text { (months) }\end{array}$ \\
\hline 1 & 40 & $\mathrm{M}$ & S 1 & 7 & 120 & 500 & 12 \\
\hline 2 & 35 & $\mathrm{~F}$ & S 3 & 10 & 90 & 200 & 6 \\
\hline 3 & 58 & $\mathrm{~F}$ & S 4 & 9.5 & 95 & 80 & 28 \\
\hline 4 & 47 & $\mathrm{~F}$ & S 1 & 7.5 & 110 & 100 & 36 \\
\hline 5 & 62 & $M$ & S 3 & 8 & 70 & 300 & 24 \\
\hline 6 & 64 & $\mathrm{~F}$ & S 2 & 12 & 130 & 300 & 39 \\
\hline 7 & 51 & $\mathrm{M}$ & S 4 & 11 & 115 & 200 & 41 \\
\hline 8 & 70 & $\mathrm{~F}$ & S 3 & 10. 5 & 120 & 350 & 33 \\
\hline 9 & 75 & $\mathrm{M}$ & S 4 & 7 & 120 & 150 & 27 \\
\hline 10 & 64 & $\mathrm{~F}$ & S 3 & 8 & 60 & 100 & 6 \\
\hline 11 & 53 & $\mathrm{M}$ & S 1 & 12 & 95 & 200 & 15 \\
\hline 12 & 58 & $\mathrm{~F}$ & S 2 & 8 & 65 & 50 & 38 \\
\hline 13 & 21 & $\mathrm{~F}$ & S 5 & 9.5 & 120 & 100 & 33 \\
\hline 14 & 32 & $\mathrm{~F}$ & S 4 & 8 & 85 & 90 & 60 \\
\hline 15 & 79 & $\mathrm{~F}$ & S 2 & 10 & 120 & 250 & 37 \\
\hline 16 & 63 & $\mathrm{~F}$ & S 3 & 8.5 & 150 & 200 & 10 \\
\hline 17 & 61 & $\mathrm{~F}$ & S 2 & 9 & 130 & 250 & 49 \\
\hline 18 & 70 & $\mathrm{~F}$ & S 1 & 11 & 180 & 500 & 51 \\
\hline 19 & 28 & $\mathrm{~F}$ & S 4 & 9.5 & 90 & 200 & 26 \\
\hline 20 & 62 & M & S 3 & 12 & 160 & 500 & 9 \\
\hline 21 & 65 & $M$ & S 4 & 10 & 105 & 200 & 57 \\
\hline 22 & 27 & $M$ & S 5 & 8 & 90 & 50 & 41 \\
\hline 23 & 46 & $M$ & S 2 & 10. 5 & 70 & 300 & 25 \\
\hline 24 & 71 & $\mathrm{~F}$ & S 1 & 10 & 85 & 250 & 19 \\
\hline 25 & 49 & $M$ & S 1 & 13. 5 & 135 & 350 & 17 \\
\hline 26 & 61 & $M$ & S 3 & 8 & 90 & 50 & 23 \\
\hline 27 & 57 & $M$ & S 2 & 12. 5 & 115 & 150 & 55 \\
\hline 28 & 62 & $M$ & S 1 & 7 & 75 & 200 & 8 \\
\hline 29 & 59 & $\mathrm{~F}$ & S 4 & 10 & 80 & 150 & 32 \\
\hline 30 & 67 & $\mathrm{~F}$ & S 5 & 15 & 145 & 400 & 14 \\
\hline
\end{tabular}

M: Male, F: Female, Max: Maximum. 


\section{RESULTS}

Pre- and postoperative neurological function is listed in Table III. Wide resections were performed in 26 patients $(86.7 \%)$ and margin resections were performed in 4 patients (13.3\%). Twenty-two patients presented with sacrococcygeal pain preoperatively (16 tumors involved S3 level or upper vertebral and 6 tumors were lower than S3 level). Of these 22 patients,

Table II: The Clinical Characteristics of 30 Patients

\begin{tabular}{|c|c|}
\hline Clinical characteristics & Values \\
\hline Male & $13(43.3)$ \\
\hline Female & $17(56.7)$ \\
\hline Sacrococcygeal pain & $22(73.3)$ \\
\hline Urinary or bowel dysfunction & $10(33.3)$ \\
\hline Lower limb numbness & $7(23.3)$ \\
\hline Chordoma & $21(70)$ \\
\hline Schwannoma & $5(16.7)$ \\
\hline Giant cell tumor of bone & $4(13.3)$ \\
\hline Upper involved level of S3 or proximal part & $20(66.7)$ \\
\hline Upper involved level of S4 or distal end & $10(33.3)$ \\
\hline $\begin{array}{l}\text { The maximum diameters of } \\
\text { tumors }>\text { or }=9 \mathrm{~cm}\end{array}$ & $18(60 \%)$ \\
\hline The maximum diameters of tumors $<9 \mathrm{~cm}$ & $12(40 \%)$ \\
\hline Postoperative surgical-related complications & $6(30)$ \\
\hline Wound infection & $3(10)$ \\
\hline Wound bleeding (ml) & $2(6.7)$ \\
\hline Subcutaneous hematoma & $1(3.3)$ \\
\hline Tumor recurrence & $9(30)$ \\
\hline Mean age (years) & 55.2 \\
\hline Mean follow-up time (months) & 29 \\
\hline Mean occlusion time (minutes) & 69 \\
\hline Mean tumors diameters $(\mathrm{cm})$ & 9.7 \\
\hline Mean operation time (minutes) & 107.1 \\
\hline Mean intraoperative hemorrhage (ml) & 224.0 \\
\hline
\end{tabular}

$16(72.7 \%)$ reported improvement, 4 (18.2\%) reported same, and $2(9.1 \%)$ reported worse postoperatively. Among the 10 patients that presented preoperatively with urinary or bowel dysfunction (7 tumors involved S3 level or upper vertebral and 3 tumors were below S3), these symptoms improved in 6 patients (60\%), remained the same in $3(30 \%)$ patients, and were worse in 1 (10\%) patient at the postoperative follow-up. Three patients experienced new urinary or bowel dysfunction postoperatively. Five patients $(71.4 \%)$ claimed that lower limb numbness improved, two patients $(28.6 \%)$ claimed no changed, and no one complained of numbness or other new symptoms worsening. No patients received blood transfusion or other blood products intraoperatively or after surgery. Six patients (30\%) experienced surgery-related complications (3 wound infection, 2 wound bleeding, and 1 groin subcutaneous hematoma). All of these complications rapidly recovered with appropriate antibiotics along with meticulous dressings and pressure dressings. No tumors recurred in the schwannoma patients (Figure $3 \mathrm{~A}-\mathrm{C}$ ) during the follow-up period. Among the 21 chordomas (Figure 4A-D), 8 tumors recurred during the follow-up period. One giant cell tumor recurred (Figure $5 A-C$ ) two years after surgery.

\section{DISCUSSION}

This study reports our experience in managing sacrococcygeal region giant tumors. According to our clinical records, chordomas are the most common giant malignant tumors in the sacral region. Chordomas are the fourth most common malignant neoplasms originating from bone and have an incidence of less than 0.1 per 100,000 people per year (13). They arise from remnants of the embryonic notochord and occur exclusively within the axial skeleton (5). These tumors occur most commonly within the sacrum (50-60\%), followed by the spheno-occipital vertebrae (25-30\%), cervical region (10\%) and thoracolumbar vertebrae (5\%) (2). Patients often present with advanced disease owing to the vague and indolent symptoms produced by these slow-growing tumors deep within the pelvis (4). Giant cell tumors (GCTs) of the bone are expansile osteolytic tumors in young adults that usually occur at the end of the long bones. Although only $6 \%$ of GCTs occur in the sacrum, GCTs are the second most common type of primary tumor involving bone in the sacrum (1). Giant sacral schwannoma is rare. Due to the probability for regional expansion into the intrasacral space, and the slow growth rate of these tumors, symptoms develop late in the course of the disease when the tumor has become very large (6).

Table III: The Clinical Symptoms Changed Postoperatively

\begin{tabular}{lcccc}
\hline Symptoms & Preoperative & \multicolumn{3}{l}{ Follow-up } \\
\hline Sacrococcygeal pain & & Improved & Same & Worse \\
\hline Urinary or bowel dysfunction & 22 & $16(72.7 \%)$ & $4(18.2 \%)$ & $2(9.1 \%)$ \\
\hline Lower limb numbness & 10 & $6(60 \%)$ & $3(30 \%)$ & $1(10 \%)$ \\
\hline
\end{tabular}


Pelvic anatomy provides a huge space for tumors to grow without any symptoms. Once finally diagnosed, the bigger the size of tumor, the more difficult the surgical intervention. Intraoperative hemorrhage is one of the most challenging and life-threatening complications of such surgeries. A few studies have reported that blood loss can exceed $10,000 \mathrm{ml}$ $(8,20)$. Additionally, intraoperative bleeding impacts surgeons' clear vision of the surgical area. Several thousand milliliters of blood loss is serious but usually does not cause death as it can be compensated with rapid blood transfusion. Nonetheless, this magnitude of blood loss may cause some complications (e.g., anemia, blood dissemination diseases, and coagulation disorders), and will greatly increase the cost of treatment. Controlling intraoperative blood loss is an imperative for surgeons as they manage of the sacrococcygeal giant tumors.

Recently, several papers $(7,9,12,15,16,18)$ claimed that occlusions of the lower abdominal aorta can effectively control intraoperative hemorrhage. $\mathrm{Mi}$ et al. (9) reported that occluding the abdominal aorta with a balloon-dilation catheter effectively reduced intraoperative hemorrhage (blood loss volume was only $100-200 \mathrm{ml}$ ) and therefore assisted surgeons in the complete and safe resection of upper sacral tumors. Yang et al. (16) also reported that after occluding the abdominal aorta, intraoperative hemorrhage was dramatically less; their average blood loss was only $280 \mathrm{ml}$ (range, 200-600
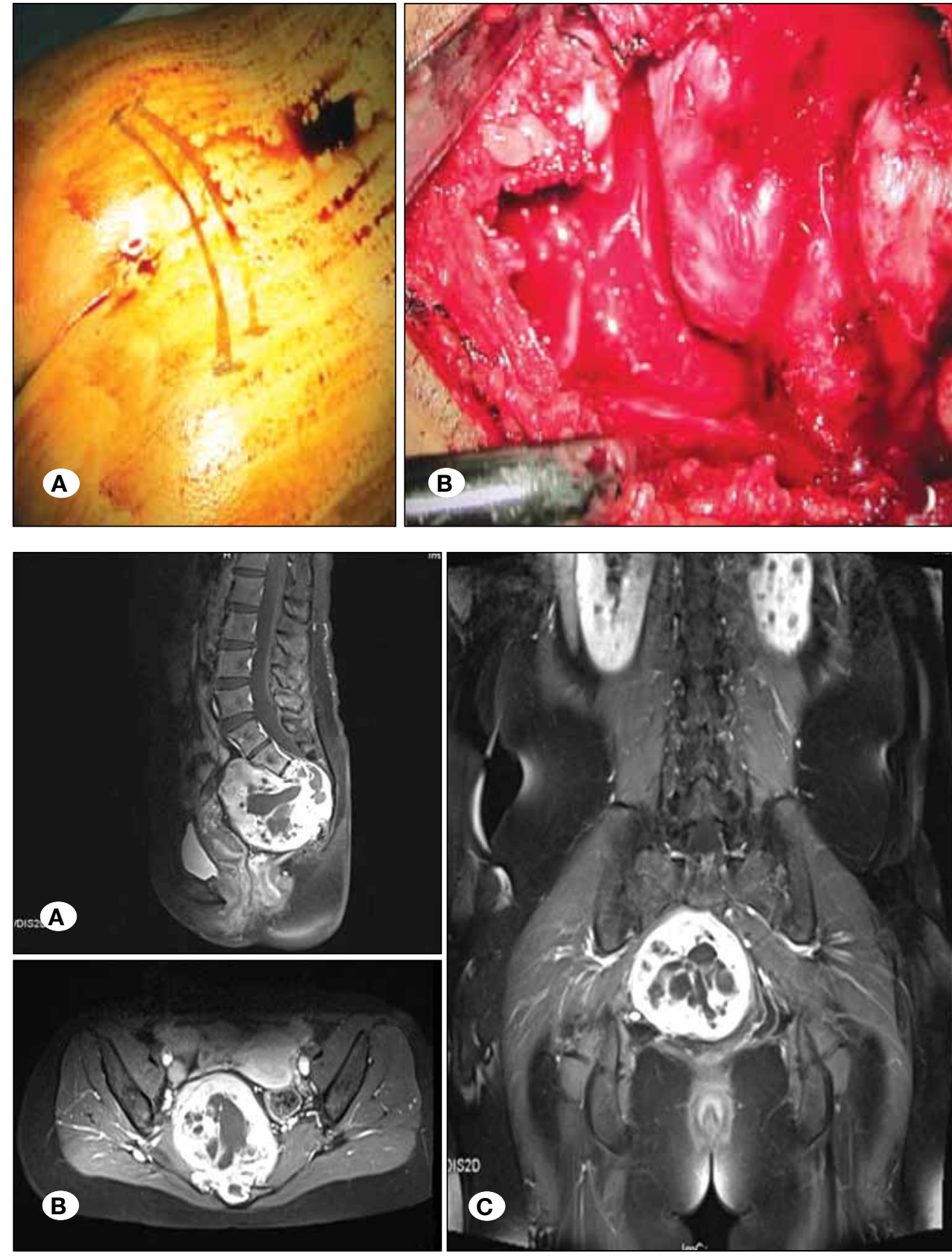

Figure 2: A "comma" incision was marked in the sacral region (A) and the upper of tumor was exposed and resected at first (B).
Figure 3: A 62-year-old male presented with sacrococcygeal pain and urinary dysfunction for three months. Sagittal MRI showed (A) the giant tumor was at the S3 level and extended from the sacral region to the pelvic cavity. Axial (B) and coronal MRI scans (C) showed that the tumor was mainly located to the right of midline and that some hypointensity signals were located in the centre of tumor. The tumor was identified as schwannoma, which was confirmed by pathological examination postoperatively. 

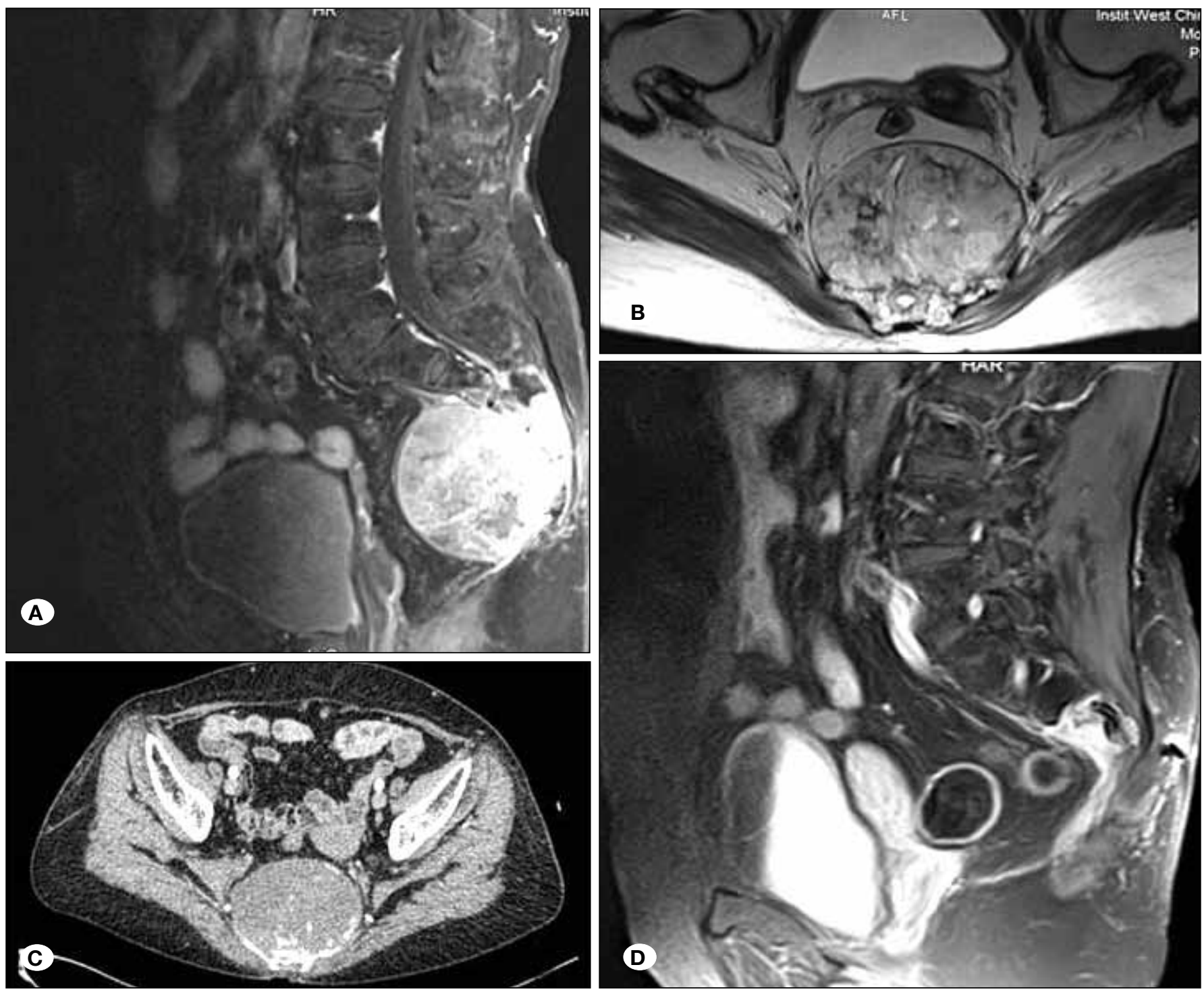

Figure 4: A 51-year-old male presented with urinary and bowel dysfunction for one month. Preoperative MRI (A, B) showed that the tumor was giant and involved the S4 level and pelvic cavity. CT (C) showed coccygeal erosion. The tumor was widely resected, as confirmed by postoperative MRI (D) scan. This tumor was chordoma.

$\mathrm{ml})$. Our study was similar to these studies in terms of blood loss; the mean intraoperative blood loss of our patients was only $224.0 \mathrm{ml}$ (range, 50-500 ml).

The literature also shows that occluding the abdominal aorta can assisted surgeons in clearly identifying the surgical margin and neighboring sacral nerves around the tumor $(7,9)$. Luo et al. (7) reported that en-bloc resection was performed in $93.3 \%$ patients in balloon-occluding group while the conventional therapy group was only $78.2 \%$. The balloon-occluding group had significantly shorter mean operating time, lower blood loss, lower blood transfusion, and lower postoperative drainage volume than the conventional therapy group (7). Our study used the technique of balloon occlusion of the lower aorta as well as electrophysiological monitoring to guarantee that the surgery was easier and safer. Wide resection was performed in most of our patients (86.7\%). The sacral nerves were better protected and the urinary or bowel function was maximally reserved. Ten patients presented preoperatively with urinary or bowel dysfunction with the sacral nerves involved. Urinary or bowel dysfunction seemed more commonly encountered in cases in which the tumor impacted upper sacral. Among these 10 patients, 7 tumors (70\%) involved the upper sacral (S3 or upper vertebral) and the rest involved the lower sacral (S4 and S5). The S2 and S3 sacral nerves were most possibly preserved, because these nerves control the urethra and anal sphincter. This result has been confirmed in multiple studies $(3,14)$. If the two $S 2$ nerve roots are preserved, approximately half of patients are likely to retain bowel and bladder function. However, bowel and bladder function are lost if only the unilateral S2 nerve root is spared (3). Unilateral resection of the sacral roots or preservation of at least one S3 nerve root 


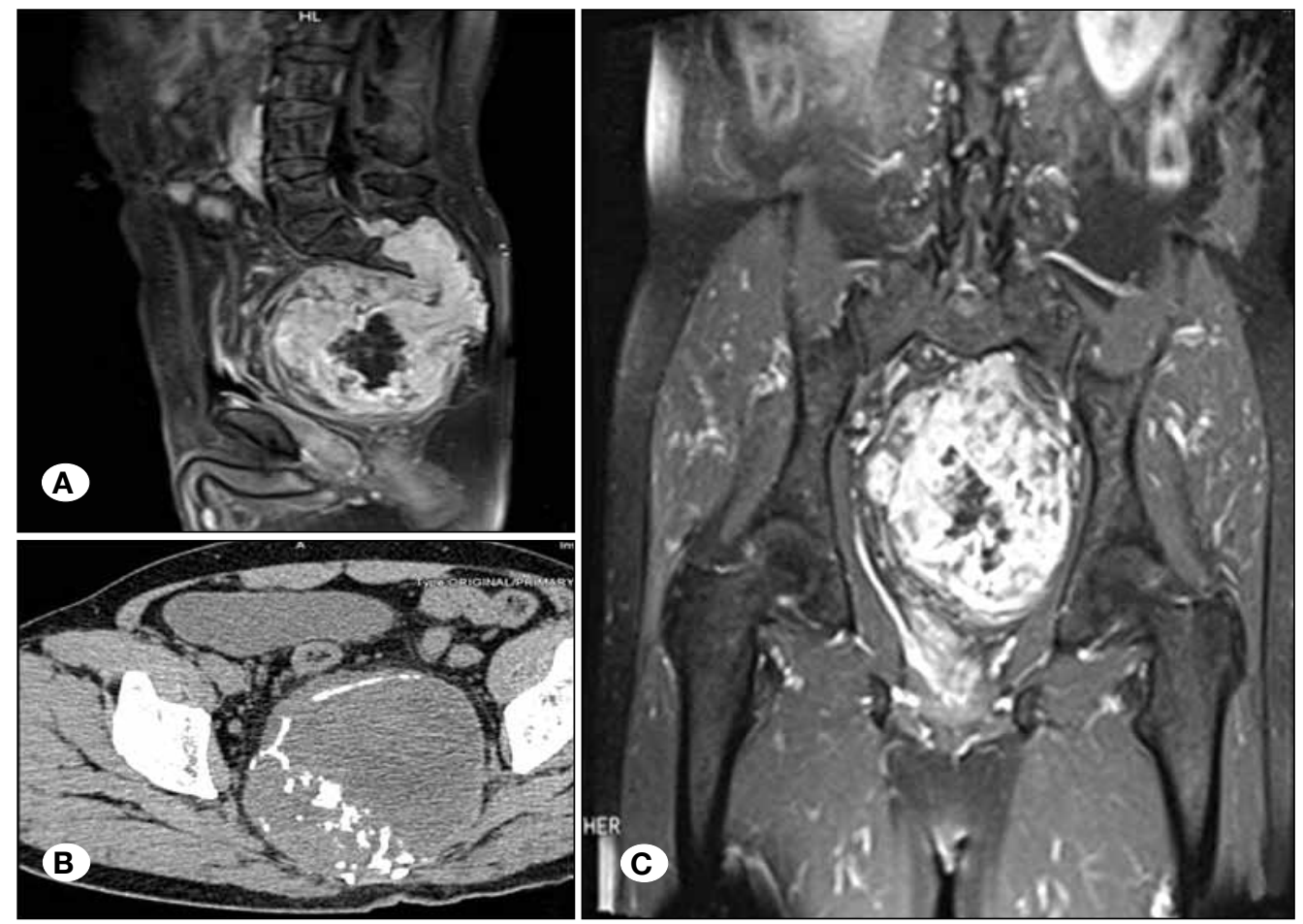

Figure 5: A 71-year-old female complained of urinary and bowel dysfunction for six months. MRI (A, C) and CT (B) showed the giant tumor located at the midline and involving the $\mathrm{S} 1$ level. There was some necrosis with a low intensity signal located in the centre of tumor. The tumor was giant cell tumor of bone. upon bilateral resection has been found to preserve bowel and bladder function in the majority of patients (14). Through surgical decompression of the sacral nerve roots, 6 patients $(60 \%)$ recovered sphincter function. However, when the nerve roots were surrounded by tumors, it was difficult to avoid damaging them. Compared with preoperative neurological dysfunction, one patient presented worse and three cases presented new sphincter dysfunction postoperatively. Sacrococcygeal pain is the most common symptom with these tumors and lesions involving upper of S4 level seemed more likely to present with pain. Compression and involvement of the sacral nerves may be the reason. Surgical decompression of nerves can effectively relieve patients' pain. Sixteen patients' $(72.7 \%)$ pain symptoms were relieved after surgery in our study.

An animal study has confirmed that internal balloon techniques may damage the vascular wall, but the damage is not serious (11). Our study and some others $(7,9,12,15,16,18)$ did not observe any vascular complications (except one patient in our study experienced occurred groin subcutaneous hematoma for incorrect compression of the puncture site). Typically, the longer the occlusion, the more complications. No consensus has been reached about a safe and conservative occlusion duration. Some studies $(15,16,18)$ have suggested that a single continuous occlusion duration should be less than 60 minutes to prevent causing arterial wall injury, ischemic necrosis of distal limb, organ injury, or multiple organ dysfunctions. Occlusion should be intermittent to restore blood flow for at least 10-15 minutes when the operating duration lasts too long. Another study (9) suggested that a safe balloon occlusion time should not be more than 90 minutes. Our experience was that a single continuous occlusion duration should be less than 60 minutes. Blood flow should be restored for 10 minutes for each 30 minutes of occlusion when the occlusion time was longer than 60 minutes. There are five notes when using this method: (1) Vascular diseases, coagulation disorders, and pelvic organ ischemia should be excluded preoperatively. (2) The balloon must be retained inferior of the bilateral renal artery and usually located at the L3-4 level. (3) It is best to use a heparin solution to fill the balloon and not use air to avoid air embolism when the balloon is ruptured (4). A volume of 250 $\mathrm{ml}$ of the heparin solution should be injected into the blood vessel to prevent thrombosis when the tumor is removed (5). Pressure dressing should be applied to the puncture site and urine volume and circulation in the lower limbs should be closely monitored for 24 hours after surgery. Although there no related-balloon-occlusion complications had detected in the followed up, but not all sacral region tumors were needed to managed by using of this method. The balloon-occlusion was only used when it matched the following criterias: (1) tumors are large and the maximum diameter more than $6 \mathrm{~cm}$; (2) the muscle or bone is invaded by tumors and the blood supply of tumors are reached according to the MRI or DSA examinations preoperatively; (3) young patients that are looking forward to reserve the bladder function after surgery; (4) old patients that accompanied with some others diseases that less tolerated to the blood losing and longer operation times; (5) there no existed any contraindications (such as any ischemia diseases of the pelvic cavity and lower limbs, any thrombus and injure of abdominal artery and coagulation disorders ) for the abdominal balloon-occlusion.

Balloon catheter occlusion of the abdominal aorta was a very effective method in controlling intraoperative bleeding. Our study indicated that it can decrease blood loss, shorten operative time, and decrease postoperative complications. 
This method is highly beneficial for the surgical management of sacrococcygeal region giant tumors. However, limitations of our study include not having a control group, a relatively short follow-up time, and a small sample.

\section{- CONCLUSION}

Sacrococcygeal region giant tumors are challenging for neurosurgeons and intraoperative hemorrhage is often encountered. Balloon catheter occlusion of the abdominal aorta is a very effective method to reduce intraoperative hemorrhage and help surgeons manage giant tumors in the sacrococcygeal region.

\section{REFERENCES}

1. Bloem JL, Reidsma II: Bone and soft tissue tumors of hip and pelvis. Eur J Radiol 81: 3793 -3801, 2012

2. Chugh R, Tawbi H, Lucas DR, Biermann JS, Schuetze SM, Baker LH: Chordoma: The nonsarcoma primary bone tumor. Oncologist 12:1344-1350, 2007

3. Guo W, Ji T, Tang X, Yang Y: Outcome of conservative surgery for giant cell tumor of the sacrum. Spine (Phila Pa 1976) 34: $1025-1031,2009$

4. Hulen CA, Temple HT, Fox WP, Sama AA, Green BA, Eismont FJ: Oncologic and functional outcome following sacrectomy for sacral chordoma. J Bone Joint Surg Am 88:1532-1539, 2006

5. Jemal A, Siegel R, Ward E, Hao Y, Xu J, Murray T, Thun MJ: Cancer statistics. CA Cancer J Clin 58: 71-96, 2008

6. Kagaya H, Abe E, Sato K, Shimada Y, Kimura A: Giant cauda equina schwannoma: A case report. Spine 25: 268, 2000

7. Luo Y, Duan H, Liu W, Min L, Shi R, Zhang W, Tu C: Clinical evaluation for lower abdominal aorta balloon occluding in the pelvic and sacral tumor resection. J Surg Oncol 108:148-151, 2013

8. Masterson EL, Davis AM, Wunder JS, Bell RS: Hindquarter amputation for pelvic tumors: The importance of patient selection. Clin Orthop 350:187-194, 1998

9. Mi C, Lu H, Liu H: Surgical excision of sacral tumors assisted by occluding the abdominal aorta with a balloon dilation catheter: A report of 3 cases. Spine 30: E614-E616, 2005
10. Ozaki T, Liljenqvist U, Halm H, Hillmann A, Gosheger G, Winkelmann W: Giant cell tumor of the spine. Clin Orthop Relat Res 401:194 -201, 2002

11. Ozalp B, Canbaz S, Huseyinova G, Duran E: Histopathological comparison of vascular wall damage created by external cross-clamp and endoluminal balloon occlusion techniques. J Cardiovasc Surg (Torino) 50:545-553, 2009

12. Ozgiray E, Cagli S, Zileli M, Cinar C, Oran I: Occlusion of the abdominal aorta by balloon dilation catheter assisting surgical excision of a sacrum chordoma: Case report. Turk Neurosurg 19:265-268, 2009

13. Samson IR, Springfield DS, Suit HD, Mankin HJ: Operative treatment of sacrococcygeal chordoma. A review of twentyone cases. J Bone Jt Surg Am 75:1476-1484, 1993

14. Todd Jr LT, Yaszemski MJ, Currier BL, Fuchs B, Kim CW, Sim $\mathrm{FH}$ : Bowel and bladder function after major sacral resection. Clin Orthop Relat Res 397: 36-39, 2002

15. Xue-Song L, Chao Y, Kai-Yong Y, Si-Qing H, Heng Z: Surgical excision of extensive sacrococcygeal chordomas assisted by occlusion of the abdominal aorta. J Neurosurg Spine 12: 490496, 2010

16. Yang L, Chong-Qi T, Hai-Bo S, Lan Z, Tian-Fu Y, Hong D, Fu-Xing P: Appling the abdominal aortic balloon occluding combine with blood pressure sensor of dorsal artery of foot to control bleeding during the pelvic and sacrum tumors surgery. J Surg Oncol 97: 626-628, 2008

17. Yokoyama R, Beppu Y, Tobisu Ki K, Moriya Y, Uchiyama K, Kito M, Umeda T, Hasegawa T, Shimoda T: A multidisciplinary approach to the treatment of malignant pelvic bone tumors: Results with eight consecutive patients. J Orthop Sci 5:449 456, 2000

18. Zhang L, Gong Q, Xiao H, Tu C, Liu J: Control of blood loss during sacral surgery by aortic balloon occlusion. Anesth Analg 105:700-703, 2007

19. Zhang ZY, Fu CF, Yang YX, Wang LQ, Cui Y, Liu Y: Long-term outcomes following en bloc resection for sacral tumor: $A$ retrospective analysis of 93 cases. Orthopedics 34:403 -407 , 2011

20. Zileli M, Hoscoskun C, Brastianos P, Sabah D: Surgical treatment of primary sacral tumors: Complications associated with sacrectomy. Neurosurg Focus 15:E9, 2003 